\title{
Non-contrast magnetic resonance venography as a preoperative imaging modality for refractory venous malformation: a case description
}

\author{
Yin-Chen Hsu ${ }^{1,2} \wedge$, Yao-Kuang Huang ${ }^{2,3}$, Yuan-Hsi Tseng ${ }^{2,3}$, Pang-Yen Chen ${ }^{4,5}$, Chao-Ming Wu ${ }^{2,6}$, \\ Chien-Wei Chen ${ }^{1,2,7}$
}

${ }^{1}$ Department of Diagnostic Radiology, Chang Gung Memorial Hospital Chiayi Branch, Puzi; ${ }^{2}$ Chang Gung University College of Medicine, Taoyuan; ${ }^{3}$ Division of Thoracic and Cardiovascular Surgery, Wound Center and Plastic Surgery, Chang Gung Memorial Hospital Chiayi Branch, Puzi; ${ }^{4}$ Department of Emergency Medicine, Mackay Memorial Hospital, Taipei; ${ }^{5}$ Institute of Environmental and Occupational Health Sciences, National Yang-Ming University, Taipei; ${ }^{6}$ Division of Plastic and Reconstructive Surgery, Wound Center and Plastic Surgery, Chang Gung Memorial Hospital Chiayi Branch, Puzi; ${ }^{7}$ Institute of Medicine, Chung Shan Medical University, Taichung

Correspondence to: Chien-Wei Chen, MD, PhD. Department of Diagnostic Radiology, Chang Gung Memorial Hospital Chiayi Branch, Chang Gung University College of Medicine, No.6, Sec. W., Jiapu Rd., Puzi City, Chiayi County. Email: chienwei33@gmail.com.

Submitted Jun 26, 2021. Accepted for publication Sep 29, 2021.

doi: 10.21037/qims-21-670

View this article at: https://dx.doi.org/10.21037/qims-21-670

\section{Introduction}

Venous malformations (VMs) are the most common vascular anomalies caused by developmental defects during the embryonic stage of life. Although the lesions are present at birth, they remain clinically undetectable until later in life and tend to grow under appropriate conditions, causing marked morbidity (1). Although sclerotherapy is one of the most effective treatments for VMs, treatment efficacy varies with the lesion type. Therapy-refractory VMs may expand and thus require more elaborate interventions (2). Enhanced magnetic resonance venography (MRV) and computed tomography venography (CTV) (with contrast) are used for further assessment of the characteristics of lesions and surrounding structures (3). If the lesion is complicated, non-contrast MRV (NC-MRV) aids in treatment planning; the technique exploits differences in the vascular signal intensities over the cardiac cycle and requires no contrast agent (4). Here, we present a report on a patient with a VM treated by percutaneous n-butyl cyanoacrylate (NBCA) embolization followed by excision. The principles of NC-MRV and its application to determine the lesion structure in a patient with complicated venous disease are described. Finally, we evaluate the short-term outcomes of the treatment.

\section{Case presentation}

The study was approved by the Institutional Review Board (IRB) of Chang Gung Medical Foundation (No. 201900448B0), and all procedures involving human participants were performed in accordance with the ethical standards of the institutional and/or national research committee(s) and the Helsinki Declaration (as revised in 2013). Written informed consent was obtained from the patient for publication of this case report and accompanying images. A copy of the written consent is available for review by contacting the editorial office of this journal.

A 40-year-old woman presented with isolated right ankle swelling and pain that had progressed over 1 year. She had not experienced any ankle weakness, paresthesia, pallor, or coldness. Doppler ultrasonography revealed an ill-defined, heterogeneous, hypoechoic cleft surrounded by anechoic fluid and small varicose veins. A poorly circumscribed

^ ORCID: 0000-0003-4483-486X. 

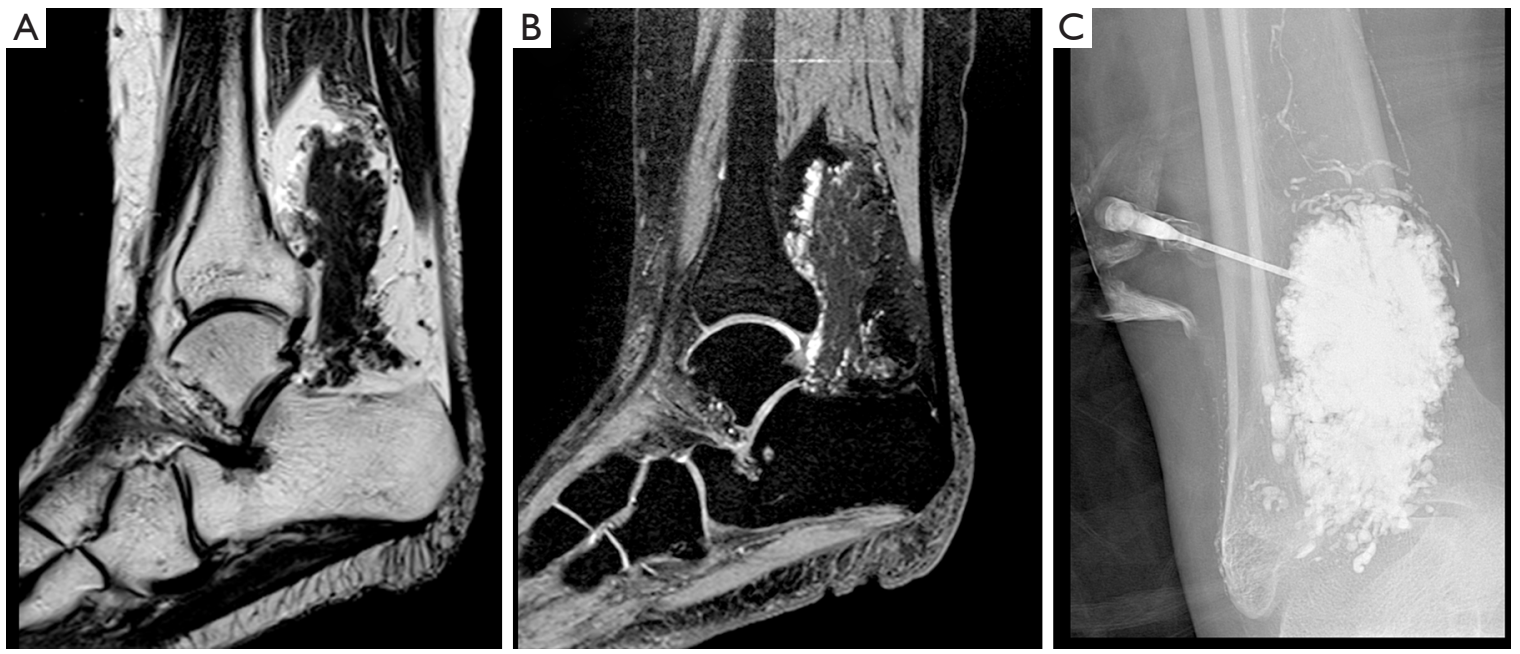

Figure 1 Preoperative magnetic resonance image of the lesion and a radiograph taken during the sclerotherapy. (A,B) T1 and T2 fatsuppressed sagittal images show a mass-like vascular malformation of the lower extremity. Noted the T2 hyperintense signal with internal septation and microcystic structures. (C) The postsclerotherapy radiograph shows a vascular tangle. Note the fine venous drainage appears as dotty and fragmented opacification.

mass-like lesion was evident in the posterior right ankle on magnetic resonance T1 (Figure 1A) and T2 fat-suppressed images (Figure 1B); the lesion measured $7 \mathrm{~cm}$ and exhibited microcystic structures and internal septation. After multidisciplinary consultation, a VM was diagnosed. Prior to planned transarterial sclerotherapy, digital subtraction angiography was performed which revealed no opacification of the abnormal vascular structure. Subsequently, sclerotherapy was performed using an ethanol-lipiodol mixture (1:3 ratio) via a direct-puncture approach. The technical end point of the procedure was the apparent influx of fluid into the connecting vessels of the lesion projection (Figure 1C).

One month after treatment, the patient complained of worsening pain and swelling of the right ankle. Follow-up radiography revealed near-total washout of the sclerosant. As the symptoms indicated that surgical resection was required, 3-dimensional (3D)-rendered images were needed to assist with preoperative planning. As no artery fed the lesion directly, flow assessment was difficult via enhanced CTV or MRV (with a contrast agent). We therefore performed NC-MRV, which revealed a residual ill-defined lesion in the right ankle, connected to small collateral veins, suggestive of a VM with venous drainage (Figure $2 A$ ). Direct-puncture embolization was performed using a 1:3 mixture of NBCA and lipiodol. Postembolization NCMRV showed no sign of the VM (Figure 2B), indicating that complete lesion casting was achieved (Figure $3 A$ ). One week later, we removed the lesion via surgical excision (Figure $3 B$ ). The patient was satisfied with the treatment. At the 1-year follow-up, Doppler ultrasonography revealed near-complete lesion regression.

\section{Discussion}

The management of VMs differs greatly with the lesion type. Sclerotherapy yields fairly satisfactory results for treating well-circumscribed VMs lacking venous drainage (5). However, our initially incorrect definition of the lesion was associated with a limited therapeutic effect, and thus surgical resection was required. Contrast-enhanced MRV and CTV are essential when planning surgery; however, if a lesion lacks a feeding artery, optimal contrast enhancement is difficult. In such a case, NC-MRV can reveal isolated, vascular structures (arteries or veins) and facilitate reasonable lesion mapping to aid in preintervention assessment and surgical planning (Video 1).

NC-MRV images were acquired using a $1.5 \mathrm{~T}$ magnetic resonance imaging (MRI) scanner (Philips Ingenia; Philips Healthcare, Best, the Netherlands). The patient underwent imaging and contemporaneous electrocardiography while supine. The abdominal aortic flow was quantitatively determined to identify appropriate trigger times for the systolic and diastolic phases. Images of the venolymphatic 

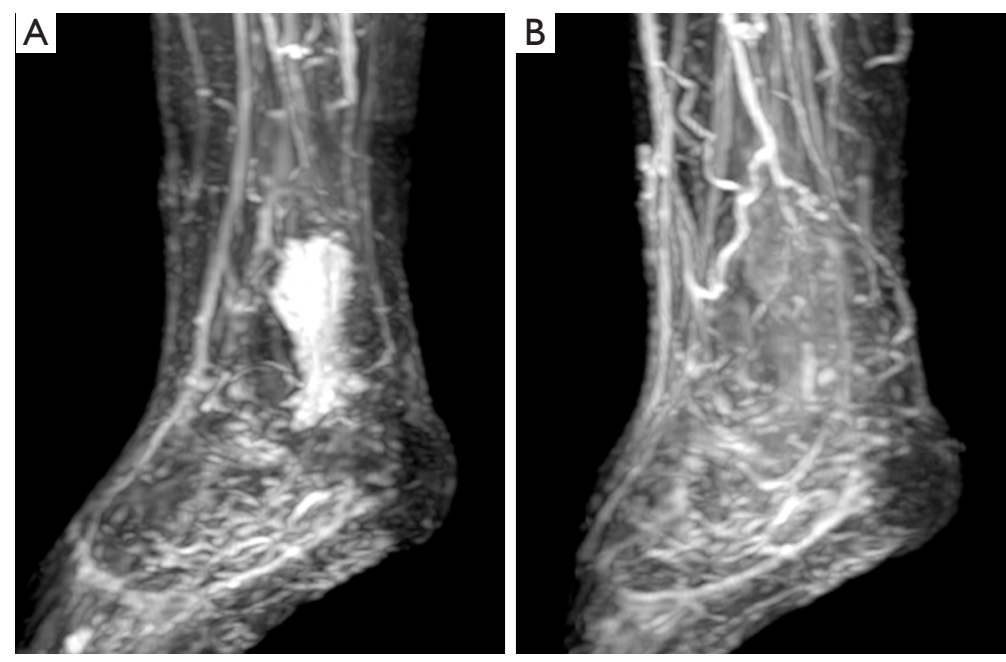

Figure 2 Comparison of pre-embolization and postembolization non-contrast magnetic resonance venography (NC-MRV) images. (A) Pre-embolization NC-MRV reveals an amorphous venous lesion in the posterior right ankle, connected to small collateral veins. (B) Postembolization NC-MRV shows exclusion of the venous malformation with patency of the deep and superficial venous return. Note the lymphedema appears as subcutaneous ground-glass signals.
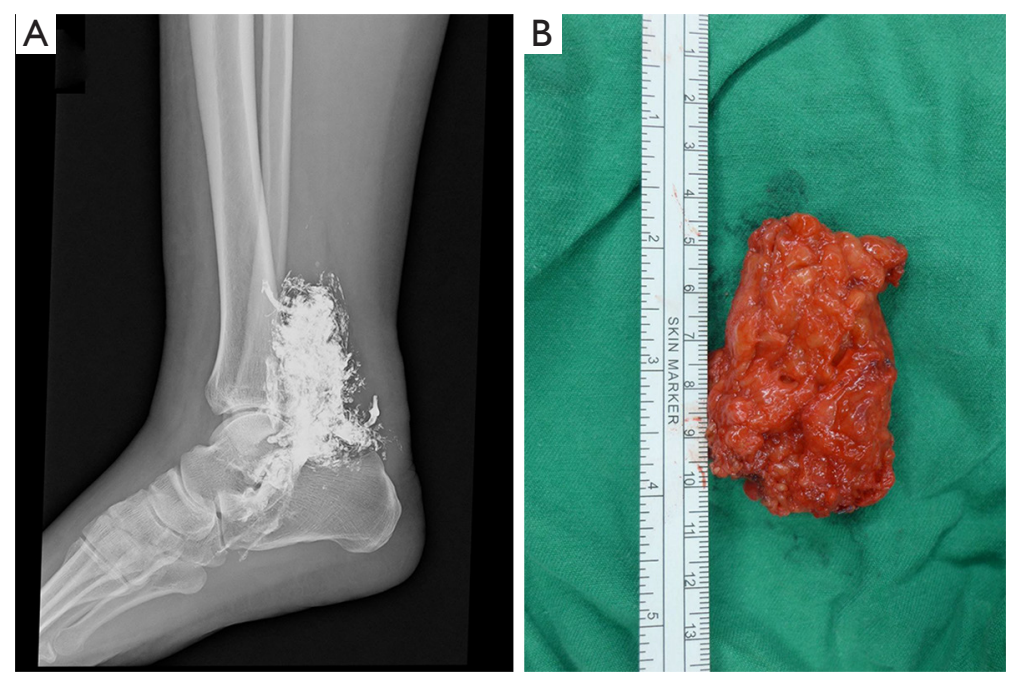

Figure 3 A side-by-side comparison of the postembolization radiograph and the resectate. (A) A postembolization radiograph of the right ankle shows complete casting of the lesion cavity, with minimal egress of injection between the deep fascia layers. (B) An intraoperative photograph shows a gross specimen of glue-filled venous malformation, which was resected en bloc. The lesion measured $5.0 \mathrm{~cm} \times 4.0 \mathrm{~cm} \times$ $3.2 \mathrm{~cm}$.

structures were acquired using a 3D turbo spin-echo, shorttau inversion recovery (STIR) sequence acquired during the systolic phase. STIR imaging facilitated background suppression of fatty and bony tissue, and the arteries were dark during cardiac systole (6). Thus, the $3 \mathrm{D}$ data set contained only the venolymphatic structures.
NC-MRV revealed an extensive VM with venous drainage; the substantial venous outflow explained the sclerosant washout. Stand-alone excision is associated with significant technical challenges, including intraoperative bleeding, poor margin delineation, and infiltrative lymphedema. We considered NBCA embolization 


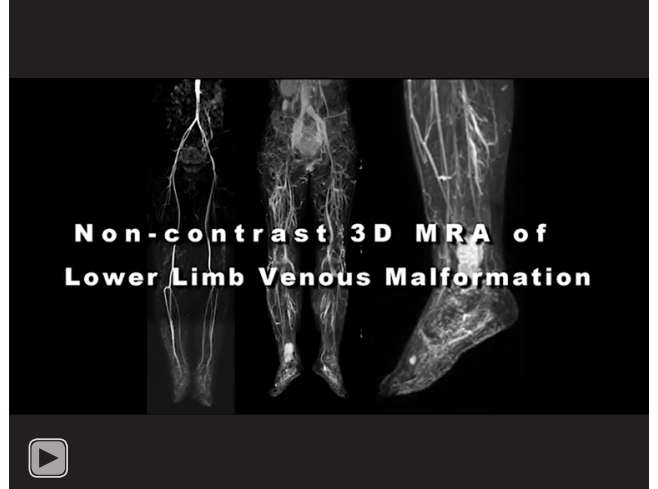

Video 1 The video demonstrates the 3-dimensional noncontrast magnetic resonance venography of lower limb venous malformation.

followed by excision, as described previously (7). NBCA tightly seals the lesion cavity and thus completely blocks outflow, transforming a blood-filled VM into a hemostatic mass. After performing NBCA embolization, the surgeon reported that the lesion margin was well-defined and removed the lesion entirely with minimal blood loss.

NC-MRV exhibits certain drawbacks, the first of which is false-positive indications when vascular complexes are suffused with different inflows from various directions (8). Second, NC-MRV imaging is time-consuming and thus inappropriate for patients with critically ill or claustrophobia. Finally, NC-MRV is expensive and therefore not widely available. We hope that presenting these findings may encourage future research.

In conclusion, NC-MRV is a useful tool for assessing complicated VMs that lack feeding arteries. For the planning of surgical interventions, percutaneous NBCA embolization followed by lesion excision is a safe and effective strategy.

\section{Acknowledgments}

We would like to thank Textcheck for English revision of this manuscript.

Funding: This study was supported by the Chang Gung Memorial Hospital (CMRPG6K0341 and CMRPG6H0651). Chang-Gung Memorial Hospital was responsible for the project management, data management, statistics, and study registration and was involved in the preparation of the manuscript.

\section{Footnote}

Conflicts of Interest: All authors have completed the ICMJE uniform disclosure form (available at https://dx.doi. org/10.21037/qims-21-670). The authors have no conflicts of interest to declare.

Ethical Statement: The authors are accountable for all aspects of the work in ensuring that questions related to the accuracy or integrity of any part of the work are appropriately investigated and resolved. The study was approved by the Institutional Review Board (IRB) of Chang Gung Medical Foundation (No. 201900448B0), and all procedures involving human participants were performed in accordance with the ethical standards of the institutional and/or national research committee(s) and the Helsinki Declaration (as revised in 2013). Written informed consent was obtained from the patient for publication of this case report and accompanying images. A copy of the written consent is available for review from the editorial office of this journal.

Open Access Statement: This is an Open Access article distributed in accordance with the Creative Commons Attribution-NonCommercial-NoDerivs 4.0 International License (CC BY-NC-ND 4.0), which permits the noncommercial replication and distribution of the article with the strict proviso that no changes or edits are made and the original work is properly cited (including links to both the formal publication through the relevant DOI and the license). See: https://creativecommons.org/licenses/by-nc-nd/4.0/.

\section{References}

1. Richter GT, Friedman AB. Hemangiomas and vascular malformations: current theory and management. Int J Pediatr 2012;2012:645678.

2. Uller W, El-Sobky S, Alomari AI, Fishman SJ, Spencer SA, Taghinia AH, Chaudry G. Preoperative Embolization of Venous Malformations Using n-Butyl Cyanoacrylate. Vasc Endovascular Surg 2018;52:269-74.

3. Greene AK, Alomari AI. Management of venous malformations. Clin Plast Surg 2011;38:83-93.

4. Hsu YC, Huang YK, Hsu LS, Chen PY, Chen CW. Using non-contrast-enhanced magnetic resonance venography for the evaluation of May-Thurner syndrome in patients with renal insufficiency: A case report. Medicine 
(Baltimore) 2019;98:e18427.

5. Dompmartin A, Vikkula M, Boon LM. Venous malformation: update on aetiopathogenesis, diagnosis and management. Phlebology 2010;25:224-35.

6. Huang YK, Tseng YH, Lin CH, Tsai YH, Hsu YC, Wang SC, Chen CW. Evaluation of venous pathology of the lower extremities with triggered angiography noncontrast-enhanced magnetic resonance imaging. BMC Med Imaging 2019;19:96.

7. Chewning RH, Monroe EJ, Lindberg A, Koo KSH,

Cite this article as: Hsu YC, Huang YK, Tseng YH, Chen PY, Wu CM, Chen CW. Non-contrast magnetic resonance venography as a preoperative imaging modality for refractory venous malformation: a case description. Quant Imaging Med Surg 2022;12(3):2153-2157. doi: 10.21037/qims-21670
Ghodke BV, Gow KW, Javid PJ, Jinguji TM, Perkins JA, Shivaram GM. Combined glue embolization and excision for the treatment of venous malformations. CVIR Endovasc 2018;1:22.

8. Chen CW, Ting H, Chen PY, Weng JC, Hsu YC, Wang SC, Tseng YH, Huang YK. Usefulness of triggered noncontrast-enhanced magnetic resonance angiography in assessing lower extremity venous disease. Medicine (Baltimore) 2021;100:e25809. 